CASE REPORT

\title{
Thalidomide for treatment of severe intestinal bleeding
}

\author{
J Bauditz, G Schachschal, S Wedel, H Lochs
}

Gut 2004;53:609-612. doi: 10.1136/gut.2003.029710

\begin{abstract}
Apart from its anti-inflammatory activity, which has been used for the treatment of active Crohn's disease, thalidomide is also a potent inhibitor of angiogenesis. We therefore studied the effect of thalidomide in six patients with severe recurrent intestinal bleeding refractory to standard treatment (three patients with Crohn's disease (CD), three patients with obscure intestinal bleeding; mean of 56 blood transfusions within the last 24 months). Bleeding stopped within two weeks after the start of thalidomide in all patients. Haemoglobin normalised without further transfusions for the whole observation period (mean follow up 33 months) while patients needed a mean of 2.2 (CD) and 3.1 (obscure bleeding) blood units/month in the 12 months before treatment. After three months of thalidomide therapy, serum levels of vascular endothelial growth factor were strongly suppressed compared with pretreatment levels. (CD 818 (82) v 129 (86) pg/ml; obscure bleeding 264 (68) v 50 (25) $\mathrm{pg} / \mathrm{ml}$ ). All six patients reported transient fatigue. Peripheral neuropathy was observed in one patient with $C D$ after nine months and was reversible after lowering the dose to $100 \mathrm{mg}$ daily. These results indicate that thalidomide might be useful for patients with otherwise refractory intestinal bleeding.
\end{abstract}

$\mathrm{S}$ evere gastrointestinal bleeding, which cannot be clearly localised, poses a major therapeutic challenge. The most frequent cause of obscure bleeding from the small intestine in patients older than 50 years are angiodysplasias, which are predominantly found in the lower ileum. ${ }^{12}$ Therapeutic strategies in these patients range from angiographic embolisation to surgical resection, which still may not successfully stop the bleeding in some cases. Currently, there is no established medical treatment available for these patients. Hormonal therapy initially showed promising results ${ }^{3}$ which were not confirmed in a recent randomised study. ${ }^{4}$ Although angiodysplasias are considered degenerative in origin, their aetiology is still unclear. Recently, neoangiogenesis in colonic angiodysplasias was shown to be mediated by vascular endothelial growth factor (VEGF), which is strongly expressed within vessel walls. ${ }^{5}$

Crohn's disease (CD) may also be complicated by severe intestinal bleeding. Although only approximately $1-2 \%$ of patients suffer from acute massive bleeding, ${ }^{67}$ these cases present a clinical challenge. One publication reported three fatalities in a series of 21 patients with severe intestinal bleeding due to $\mathrm{CD} .{ }^{6}$ In these patients the exact bleeding site is often not identifiable and severe bleeding may recur despite intestinal resection. ${ }^{6}$ Interestingly, the occurrence of massive bleeding was not found to be associated with disease activity. ${ }^{6} 7$

In a recent study on the treatment of steroid refractory CD with thalidomide, we noticed a rapid improvement in rectal bleeding in patients with haemorrhagic diarrhoea. ${ }^{8}$ Interestingly, thalidomide not only displays anti-inflammatory activity but is also an inhibitor of angiogenesis, which is mediated by suppression of VEGF. ${ }^{9}{ }^{10}$ As increased production of VEGF is found in $C D,{ }^{11-13}$ we hypothesised that suppression of VEGF might be an effective therapy in cases of severe bleeding. We therefore treated a series of patients with severe therapy refractory intestinal bleeding related to $\mathrm{CD}$ and obscure bleeding with thalidomide.

\section{CASE SERIES}

Six patients with severe intestinal bleeding (mean of 56 blood transfusions within 24 months) resistant to endoscopic, surgical, and anti-inflammatory therapy were treated. All patients underwent extensive unsuccessful diagnostic procedures to localise the site of bleeding.

Three patients with CD (patients A-C; CD activity index (CDAI) 251 (31), CD endoscopic index of severity (CDEIS) 13.1 (1.8)) suffered from recurrent episodes of intestinal bleeding. Although all three patients had endoscopically detectable lesions, a clear bleeding site could not be identified. Bleeding episodes were not accompanied by an increase in inflammation parameters or increased clinical activity. These three patients needed a mean of 2.2 blood units/month during the last year (fig 1). Patients were started on $300 \mathrm{mg}$ thalidomide (Gruenenthal, Germany) daily, the same dose which we had used in our previous study in CD. ${ }^{8}$ Thalidomide was lowered to 50-100 mg after 6-9 months. Clinical evaluation with assessment of CDAI was performed at baseline and at least every three months. Colonoscopy with evaluation of CDEIS was performed before thalidomide and at three months.

Three further patients (patients D-F) suffered from obscure bleeding of the small intestine. Within the last 12 months, patients had between three and 11 severe bleeding episodes requiring admission to hospital and received a mean of 3.1 blood units/month (fig 1). In one patient, wireless capsule endoscopy demonstrated multiple angiodysplasias within the jejunum and ileum; in two patients capsule endoscopy had not yet been available. However, by endoscope, the source of bleeding was located to the ileum.

Although angiodysplasias of the small bowel could be visualised by wireless capsule endoscopy in only one of these patients, we regarded bleeding in all three patients as highly likely to be related to angiodysplasias. Endoscopic and capsule endoscopy studies show that the overwhelming majority of cases of obscure bleeding from the small intestine in older patients, similar to ours, is related to angiodysplasias.

In younger patients with obscure bleeding from the small intestine, the single most common lesions found are small bowel tumours. However, in patients older than 50 years, angiodysplasias are thought to cause obscure bleeding from the small intestine in $>90 \%$ of patients. ${ }^{12}{ }^{14}$ Only $6 / 207$ (3\%)

\footnotetext{
Abbreviations: CD, Crohn's disease; VEGF, vascular endothelial growth factor; CDAl, Crohn's disease activity index; CDEIS, Crohn's disease endoscopic index of severity
} 


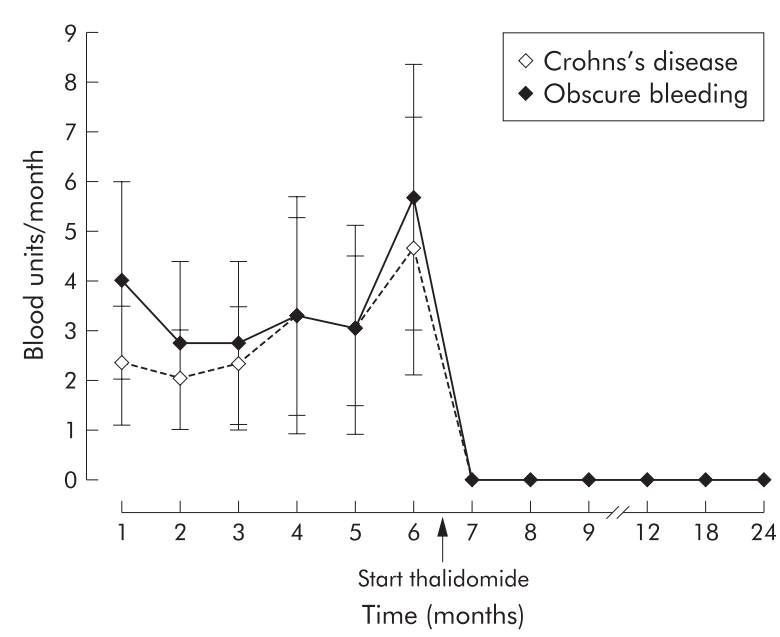

Figure 1 Number of blood transfusions/month (mean (SEM)) in patients with Crohn's disease and patients with obscure bleeding. No further transfusions were required after the start of thalidomide (follow up: 22-49 months; mean 33 months).

patients older than 50 years were found to bleed due to small bowel tumours. ${ }^{2}$ Furthermore, our patients suffered from severe bleeding, a characteristic feature of vascular malformations. ${ }^{2}$ We therefore regarded these two patientsalthough not proven-as highly suspicious of bleeding from angiodysplasias.

As the optimal dose for antiangiogenic treatment is unclear and no anti-inflammatory activity was needed in these patients, we chose low dose treatment of $100 \mathrm{mg}$ daily thalidomide to minimise toxicity in these elderly patients. After four months, thalidomide was stopped. Clinical evaluation with control of haemoglobin levels was performed at baseline and at least every four weeks.

Patients were followed for a mean of 33 months (range 2249). As thalidomide can cause peripheral neuropathy, careful neurological evaluation was performed before the start of treatment and every three months. Electromyography was performed at baseline, at the end of treatment, or when clinical symptoms possibly related to neuropathy occurred. Treatment was approved by the local ethics committee. Informed consent was obtained from all patients. Serum VEGF levels were measured in duplicate before the start of thalidomide therapy and after three months. VEGF ELISA kits were obtained from R\&D Systems (Minneapolis, Minnesota, USA). Sensitivity of VEGF ELISA is $31.2 \mathrm{pg} / \mathrm{ml}$. Values of disease activity indices and VEGF are given as mean (SEM).

\section{CASE REPORTS}

\section{Patient A}

A 34 year old man with a nine year history of CD suffered from severe recurrent intestinal bleeding which required 14 admissions to hospital within the last three years. Because of bleeding, three resections of the small intestine were performed without lasting effect. He received a total of 57 blood transfusions despite treatment with mesalazine $(3 \mathrm{~g})$, azathioprine (100 mg), and up to $100 \mathrm{mg}$ of prednisone. Within the last six months he was dependent on $20 \mathrm{mg}$ of prednisone and received 29 blood transfusions. Colonoscopy showed moderate inflammation with aphthous lesions of the rectum, and ulcers and angiodysplasias near the anastomosis. However, the bleeding site could not be identified. After the start of $300 \mathrm{mg}$ thalidomide daily, bleeding stopped within two weeks with no requirement for transfusions in the following 12 months. Thalidomide was then discontinued and bleeding recurred 13 months later. After starting retreatment $(300 \mathrm{mg}$, tapered to $100 \mathrm{mg}$ within three months), bleeding stopped again within two weeks and did not recur during the following 24 months.

\section{Patient B}

A 42 year old man had an 11 year history of CD. Despite strong immunosuppressive therapy (azathioprine $125 \mathrm{mg}$, prednisone 25-60 mg, mesalazine $3 \mathrm{~g}$ daily, and mesalazine enemas) he suffered from severe recurrent intestinal bleeding. Resection of the small intestine because of bleeding was without lasting effect. Within the last six months he had received a total of 24 units of blood. His situation was further complicated by thrombophilia of unknown origin which had led to previous repeated deep venous thromboses, pulmonary embolism, and myocardial infarction. Due to intestinal bleeding, antithrombotic therapy had to be stopped six months ago. Colonoscopy showed inflammatory lesions within the ileum and rectum with no clear bleeding lesion. After the start of thalidomide (300 mg), bleeding subsided within two weeks and antithrombotic therapy could be started. The patient is still on heparin without bleeding for the past 34 months.

\section{Patient C}

A 33 year old man with a four year history of CD treated by $3 \mathrm{~g}$ mesalazine and budesonide enemas had become dependent on $20 \mathrm{mg}$ prednisone for the past seven months. Despite treatment, he had 10-12 bloody stools daily leading to chronic anaemia (haemoglobin $6.8-8.5 \mathrm{mg} / \mathrm{dl}$ ). He refused blood transfusions. Colonoscopy showed mild ileocolitis. After the start of thalidomide (300 mg), bleeding stopped within two weeks and within three months he stabilised on three bowel movements daily and a haemoglobin level of $>13 \mathrm{mg} / \mathrm{dl}$. Discontinuation of thalidomide after 12 months resulted in recurrence of bloody diarrhoea within four weeks. After starting retreatment $(300 \mathrm{mg}$, tapered to $100 \mathrm{mg}$ within three months), bleeding stopped again within two weeks and did not recur during the following 25 months.

\section{Patient D}

A 54 year old man suffered from severe recurrent intestinal bleeding for the past three and a half years in which he received a total of 237 units of blood. During the last 12 months, bleeding episodes had led to 11 admissions to hospital where he received 85 units of blood. Despite extensive diagnostic procedures, including repeated gastroscopy, colonoscopy, enteroscopy, computed tomography of the small bowel, angiography, meckel scan, ${ }^{99 \mathrm{~m}}$ Tc labelled red blood scintigraphy during bleeding, and even laparotomy, the bleeding site could not be identified. However, from endoscopy during acute bleeding it was clear that the bleeding site was located within the small bowel. As the patient was suspected of bleeding from angiodysplasias of the small bowel, antiangiogenic treatment with thalidomide was discussed with the patient. After low dose thalidomide (100 mg daily) was started, bleeding stopped within two weeks. Although thalidomide treatment was stopped after four months, no further bleeding episode occurred during a follow up of 33 months.

\section{Patient E}

A 69 year old woman suffered from severe recurrent intestinal bleeding for the past one year, which had led to three admissions to hospital during which she received 12 units of blood. Despite repeated gastroscopy, colonoscopy, enteroscopy, meckel scan, computed tomography of the small bowel, and angiography, the bleeding site could not be identified. However, endoscopy during acute bleeding 
indicated that the bleeding site was located within the small bowel. As she was suspected of bleeding from recurrent angiodysplasias of the small bowel, antiangiogenic treatment with thalidomide was discussed with the patient. After low dose thalidomide ( $100 \mathrm{mg}$ daily for four months) was started, no further bleeding episode occurred in the following 24 months.

\section{Patient F}

A 72 year old man suffered from recurrent intestinal bleeding for the past two years which had led to four admissions to hospital. During the last year he received 14 units of blood. Despite repeated gastroscopy, colonoscopy, enteroscopy, $x$ ray of the small bowel, and ${ }^{99 \mathrm{~m}}$ Tc labelled red blood scintigraphy, the bleeding site could not be identified. Wireless capsule endoscopy demonstrated multiple angiodysplasias of the jejunum and ileum. After the start of thalidomide (100 mg daily for four months), no further bleeding episodes occurred in the following 22 months.

Treatment was well tolerated by all patients. No major side effects were seen apart from transient fatigue (which was reported by all six patients) and peripheral neuropathy, which developed in one patient (patient A) after nine months and was reversible after lowering the dose to $100 \mathrm{mg}$ daily. Haemoglobin normalised without further transfusions during treatment with thalidomide in all six patients and remained stable during a total follow up of mean 33 months (range 22-49). No transfusions were necessary during follow up (fig 1).

In CD patients, clinical activity decreased during treatment (CDAI 251 (31) before treatment, 116 (15) after three months of thalidomide). CDEIS decreased from 13.1 (1.8) before treatment to $7.9(0.9)$ after three months, $\mathrm{C}$ reactive protein decreased from 15.3 (1.8) to 6 (3.1) $\mathrm{mg} / \mathrm{l}$, and platelets changed from 333 (41) before treatment to 318 (34) after three months. Diarrhoea improved in two patients (weekly stools 27 (4.1) $v 17.7(2.9)$ ); one patient reported relief of abdominal pain. In patients with $\mathrm{CD}$, production of VEGF decreased from 818 (82) pg/ml before treatment to 129 (86) $\mathrm{pg} / \mathrm{ml}$ after three months (fig 2).

No signs of inflammation (C reactive protein, leucocytes, platelets, erythrocyte sedimentation rate) were observed in patients with obscure bleeding. In these patients, production of VEGF decreased from $264(68) \mathrm{pg} / \mathrm{ml}$ before treatment to 50 (25) $\mathrm{pg} / \mathrm{ml}$ (fig 2).

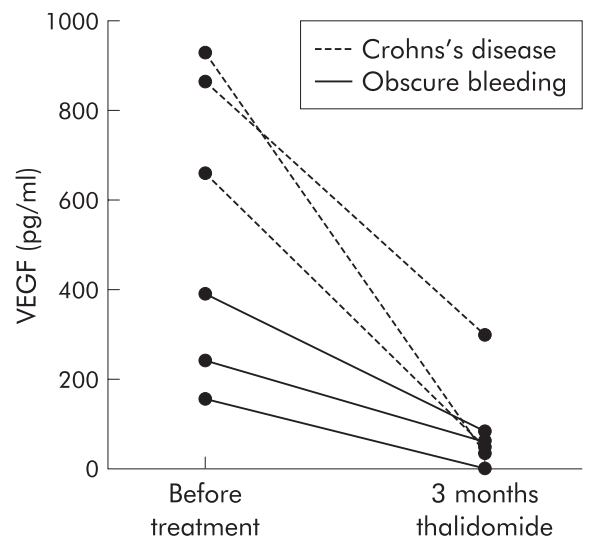

Figure 2 Serum levels of vascular endothelial growth factor (VEGF) in patients with Crohn's disease and patients with obscure bleeding. Production of VEGF decreased in all patients after three months of thalidomide therapy.

\section{DISCUSSION}

As thalidomide displays not only anti-inflammatory effects but is also a potent inhibitor of angiogenesis, ${ }^{915}$ we investigated the effectiveness of thalidomide in patients with severe therapy resistant intestinal bleeding related to CD and bleeding of obscure origin. Thalidomide proved to be highly effective in all six patients. Bleeding stopped in all patients within two weeks after initiation of treatment. While patients had required a mean of 2.7 units of blood/month in the year before the study, no further blood transfusions were necessary during treatment with thalidomide and further follow up of 28 months. This effect was reproducible by retreatment in two patients who started to rebleed after withdrawal of thalidomide.

As thalidomide suppresses production of tumour necrosis factor $\alpha$ and interleukin 12, the underlying mechanism for this clinical efficacy in patients with CD could be related to anti-inflammatory activity. ${ }^{8}$ However, during several previous bleeding episodes, we did not observe a correlation between disease activity and occurrence of bleeding, an observation which is in accordance with previous studies on severe bleeding in $\mathrm{CD}^{6}{ }^{6}$ Overall, inflammatory activity during bleeding episodes was relatively moderate in our patients. Judging inflammation primarily by increased baseline CDAI (251 (31)) may lead to an overestimation of inflammatory activity, as anaemia alone accounted for about 80-100 points overall. As the anti-inflammatory potential of thalidomide is also relatively moderate, ${ }^{816} 17$ we hypothesise that the strong clinical effect of thalidomide on intestinal bleeding was possibly related to additional mechanisms and not exclusively to its anti-inflammatory properties.

Increased production of VEGF was recently described in CD. ${ }^{11-13}$ Although the exact role of VEGF in the inflammatory process within the intestinal mucosa is currently unclear, it is conceivable that VEGF mediated effects such as increased vascular permeability and neoangiogenesis contribute to intestinal bleeding in $\mathrm{CD}{ }^{18}$ Thalidomide is an inhibitor of neoangiogenesis, an effect which is mediated by suppression of VEGF. ${ }^{10}$ We found strong suppression of VEGF after treatment with thalidomide in our patients, offering a possible explanation for its efficacy in severe bleeding in CD. However, as VEGF levels are associated with disease activity in $\mathrm{CD}^{12}{ }^{13}$ it is also possible that decreased production of VEGF in our patients was secondary to the antiinflammatory effects of thalidomide.

Clearer evidence for a direct effect of thalidomide on suppression of VEGF may be given in patients without inflammation. Thalidomide was also effective in three patients with severe bleeding from the small intestine, presumably due to angiodysplasias without signs of inflammation. As the number of previous bleeding episodes independently predicts rebleeding in patients with gastrointestinal angiodysplasias, ${ }^{4}$ and our patients had 3-11 severe bleeding episodes requiring admission to hospital within the last 12 months, they were at high risk of rebleeding. After the start of thalidomide treatment no further bleeding episode occurred during a mean follow up of 26 months. In accordance with these findings, a therapeutic effect of thalidomide on intestinal bleeding was recently reported in a patient with acute myelogenous leukaemia and angiodysplasias. ${ }^{19}$

VEGF is strongly expressed within colonic angiodysplasias. $^{5}$ As VEGF is a key angiogenic factor that is secreted by cancer cells and normal cells in response to hypoxia, ${ }^{18}$ VEGF may also be centrally involved in the pathophysiology of angiodysplasias. Local hypoxia within the intestinal wall in older patients may trigger production of VEGF, thereby contributing to formation of angiodysplasias. Animal experiments have shown that unregulated VEGF expression can 
lead to formation of disorganised fragile vessels susceptible to rupture. ${ }^{20}$ As production of VEGF decreased after treatment with thalidomide in our patients (fig 2), the observed effect on rebleeding may be related to suppression of VEGF.

Serum concentrations of VEGF in our patients were similar to those reported in CD by others ${ }^{12}{ }^{13}$ and higher than usually seen in healthy persons. ${ }^{21}$ Suppression of VEGF by thalidomide in our patients indicates that doses of 100-300 mg efficiently inhibit mediators of angiogenesis. However, the optimal dose is unclear and may even be lower. Due to its teratogenicity, thalidomide was almost completely withdrawn from human use. However, considering the severe consequences of recurrent bleeding and the minor side effects of thalidomide in several studies, ${ }^{16}{ }^{17}$ it might be considered as a therapeutic option in intestinal bleeding resistant to conventional therapy.

In conclusion, thalidomide may be a valuable therapeutic option in patients with severe gastrointestinal bleeding of different origin. Further therapeutic evaluation of thalidomide in this indication therefore seems warranted.

\section{Authors' affiliations}

J Bauditz, G Schachschal, S Wedel, H Lochs, Charité University Hospital, IVth Department of Medicine, Berlin, Germany

Parts of this work have been published in abstract form (Gastroenterology 2002;122:A194) and have been presented at the annual meeting of the American Gastroenterological Association, San Francisco, California, May 19-22, 2002.

Correspondence to: Dr J Bauditz, Universitätsklinikum Charité, IV. Medizinische Klinik und Poliklinik, Schumannstr. 20/21, 10117 Berlin, Germany; juergen.bauditz@charite.de

Accepted for publication 10 December 2003

\section{REFERENCES}

1 Gordon FH, Watkinson A, Hodgson H. Vascular malformations of the gastrointestinal tract. Best Pract Res Clin Gastroenterol 2001:15:41-58.

2 Lewis BS, Kornbluth A, Waye JD. Small bowel tumours: yield of enteroscopy. Gut $1991 ; 32: 763-5$.
3 van Cutsem E, Rutgeerts P, Vantrappen G. Treatment of bleeding gastrointestinal vascular malformations with oestrogen-progesterone. Lancet 1990;335:953-5

4 Junquera $F$, Feu $F$, Papo $M$, et al. A multicenter, randomized, clinical trial of hormonal therapy in the prevention of rebleeding from gastrointestinal angiodysplasia. Gastroenterology 2001;121:1073-9.

5 Junquera F, Saperas E, de Torres I, et al. Increased expression of angiogenic factors in human colonic angiodysplasia. Am J Gastroenterol 1999:94:1070-6.

6 Robert JR, Sachar DB, Greenstein AJ. Severe gastrointestinal hemorrhage in Crohn's disease. Ann Surg 1991;213:207-11.

7 Homan WP, Tang CK, Thorbjarnarson B. Acute massive hemorrhage from intestinal Crohn's disease. Report of seven cases and review of the literature. Arch Surg 1976;111:901-5.

8 Bauditz J, Wedel S, Lochs $\mathrm{H}$. Thalidomide reduces TNF $\alpha$ and interleukin 12 production in patients with chronic active Crohn's disease. Gut 2002;2:196-200.

9 D'Amato RJ, Loughnan MS, Flynn E, et al. Thalidomide is an inhibitor of angiogenesis. Proc Natl Acad Sci U S A 1994:91:4082-5.

10 Kenyon BM, Browne F, D'Amato RJ. Effects of thalidomide and related metabolites in a mouse corneal model of neovascularization. Exp Eye Res 1997:64:971-8

11 Griga T, May B, Pfisterer O, et al. Immunohistochemical localization of vascular endothelial growth factor in colonic mucosa of patients with inflammatory bowel disease. Hepatogastroenterology 2002;49:1 16-23.

12 Griga T, Tromm A, Spranger J, et al. Increased serum levels of vascular endothelial growth factor in patients with inflammatory bowel disease. Scand J Gastroenterol 1998:33:504-8.

13 Bousvaros A, Leichtner A, Zurakowski D, et al. Elevated serum vascular endothelial growth factor in children and young adults with Crohn's disease. Dig Dis Sci 1999;44:424-30.

14 Ell C, Remke S, May A, et al. The first prospective controlled trial comparing wireless capsule endoscopy with push enteroscopy in chronic gastrointestinal bleeding. Endoscopy 2002;34:685-9.

15 Lentzsch S, Rogers MS, LeBlanc R, et al. S-3-Amino-phthalimido-glutarimide inhibits angiogenesis and growth of B-cell neoplasias in mice. Cancer Res 2002;62:2300-5.

16 Vasiliauskas EA, Kam LY, Abreu-Martin MT, et al. An open-label pilot study of low-dose thalidomide in chronically-active, steroid-dependent Crohn's disease. Gastroenterology 1999;117:1278-87.

17 Ehrenpreis ED, Kane SV, Cohen LB, et al. Thalidomide therapy for patients with refractory Crohn's disease: An open-label trial. Gastroenterology 1999; 117:1271-7.

18 Harris AL. Hypoxia-a key regulatory factor in tumour growth. Nat Rev Cancer 2002;2:38-47.

19 Shurafa M Kamboi G. Thalidomide for the treatment of bleeding angiodysplasias. Am J Gastroenterol 2003;98:221-2.

20 Lee RJ, Springer ML, Blanco-Bose WE, et al. VEGF gene delivery to myocardium: deleterious effects of unregulated expression. Circulation 2000;102:898-901.

21 Kraft A, Weindel K, Ochs A, et al. Vascular endothelial growth factor in the sera and effusions of patients with malignant and nonmalignant disease. Cancer 1999;85: 178-87. 